\title{
The Lateral Trigger Probability function for the Ultra-High Energy Cosmic Ray showers detected by the Pierre Auger Observatory
}

The Pierre Auger Collaboration, P. Abreu ${ }^{\text {bl }}$, M. Aglietta ay , E.J. Ahn ${ }^{\text {ca }}$, I.F.M. Albuquerque ${ }^{o}$, D. Allard ${ }^{a b}$, I. Allekotte ${ }^{\mathrm{a}}$, J. Allen ${ }^{\mathrm{cd}}$, P. Allison ${ }^{\text {cf }}$, J. Alvarez Castillo ${ }^{\text {be }}$, J. Alvarez-Muñiz ${ }^{\text {bs }}$, M. Ambrosio ${ }^{\text {as }}$, A. Aminaei ${ }^{\text {bf }}$, L. Anchordoqui ${ }^{\mathrm{cm}}, \mathrm{S}$. Andringa ${ }^{\mathrm{bl}}$, T. Antičić ${ }^{\mathrm{w}}$, A. Anzalone ${ }^{\mathrm{ax}}, \mathrm{C}$. Aramo as ${ }^{\mathrm{a}}$ E. Arganda bp, F. Arqueros ${ }^{\mathrm{bp}}$, H. Asorey ${ }^{a}$, P. Assis ${ }^{\text {bl }}$, J. Aublin ${ }^{a d}$, M. Ave ${ }^{\mathrm{ak}}$, M. Avenier ${ }^{\mathrm{ae}}$, G. Avila ${ }^{\mathrm{j}}$, T. Bäcker ${ }^{\mathrm{an}}$, M. Balzer ${ }^{\mathrm{ai}}$, K.B. Barber ${ }^{\mathrm{k}}$, A.F. Barbosa ${ }^{1}$, R. Bardenet ${ }^{\text {ac }}$, S.L.C. Barroso ${ }^{r}$, B. Baughman ${ }^{\text {cf }}$, J. Bäuml ${ }^{\text {ah,aj }}$, J.J. Beatty ${ }^{\text {cf }}$, B.R. Becker ${ }^{\text {ck }}$, K.H. Becker ${ }^{\text {ag }}$, A. Bellétoile ${ }^{\text {af }}$, J.A. Bellido ${ }^{k}$, S. BenZvi ${ }^{c l}$, C. Berat ${ }^{\text {ae }}$, X. Bertou ${ }^{\mathrm{a}}$, P.L. Biermann ${ }^{\text {ak }}$, P. Billoir $^{\text {ad }}$, F. Blanco ${ }^{\text {bp }}$, M. Blanco ${ }^{\text {bq }}$, C. Bleve ${ }^{\text {ag }}$, H. Blümer $^{\text {ah,aj }}$, M. Boháčová ${ }^{\mathrm{y}, c i}$, D. Boncioli $^{\text {at }}$, C. Bonifazi $^{\text {u,ad }}$, R. Bonino ay , N. Borodai ${ }^{\text {bj }}$, J. Brack ${ }^{\text {by }}$, P. Brogueira bl, W.C. Brown ${ }^{\text {bz }}$, R. Bruijn bu, P. Buchholz ${ }^{\text {an }}$, A. Bueno ${ }^{\text {br }}$, R.E. Burton ${ }^{\text {bw }}$, K.S. Caballero-Mora ${ }^{\text {ak }}$, L. Caramete ${ }^{\text {ak }}$, R. Caruso ${ }^{\text {au }}$, A. Castellina ${ }^{\text {ay }}$, O. Catalano ${ }^{\text {ax }}$, G. Cataldi $^{\text {ar }}$, L. Cazon $^{\text {bl }}$, R. Cester ${ }^{\text {av }}$, J. Chauvin ${ }^{\text {ae }}$, S.H. Cheng ${ }^{\text {cg }}$, A. Chiavassa ${ }^{\text {ay }}$, J.A. Chinellato ${ }^{\mathrm{p}}$, A. Chou ${ }^{\text {ca,cd }}{ }^{\mathrm{N}}$ J. Chudoba ${ }^{\mathrm{y}}$, R.W. Clay ${ }^{\text {k }}$, M.R. Coluccia ${ }^{\text {ar }}$, R. Conceição ${ }^{\text {bl }}$, F. Contreras ${ }^{i}$, H. Cook ${ }^{\text {bu }}$, M.J. Cooper ${ }^{k}$, J. Coppens ${ }^{\text {bf,bh }}$, A. Cordier ${ }^{\text {ac }}$, U. Cotti $^{\text {bd }}$, S. Coutu ${ }^{\text {cg }}$, C.E. Covault ${ }^{\text {bw }}$, A. Creusot ${ }^{\text {ab,bn }}$, A. Criss ${ }^{c g}, J$. Cronin ${ }^{\text {ci }}$, A. Curutiu ${ }^{\text {ak }}$, S. Dagoret-Campagne ${ }^{\text {ac }}$, R. Dallier ${ }^{\text {af }}$, S. Dasso ${ }^{\text {d,f }}$, K. Daumiller $^{\text {ah }}$, B.R. Dawson ${ }^{\mathrm{k}}$, R.M. de Almeida ${ }^{\mathrm{p}, \mathrm{v}}$, M. De Domenico au, C. De Donato ${ }^{\mathrm{aq}, \mathrm{be}}$, S.J. de Jong ${ }^{\text {bf }}, \mathrm{G}$. De La Vega ${ }^{\mathrm{h}}$, W.J.M. de Mello Junior ${ }^{\mathrm{p}}$, J.R.T. de Mello Neto ${ }^{\text {u, I. De Mitri }}{ }^{\text {ar }}$, V. de Souza ${ }^{\text {n }}$, K.D. de Vries ${ }^{\text {bg }}$, G. Decerprit ${ }^{\text {ab }}$, L. del Peral ${ }^{\text {bq }}$, O. Deligny ${ }^{\text {aa }}$,

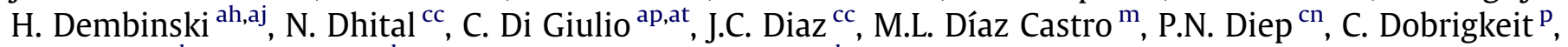
W. Docters ${ }^{\text {bg }}$, J.C. D'Olivo ${ }^{\text {be }}$, P.N. Dong ${ }^{\text {aa,cn }}$, A. Dorofeev ${ }^{\text {by }}$, J.C. dos Anjos a , M.T. Dova ${ }^{\text {e, D. D'Urso }}{ }^{\text {as }}$, I. Dutan $^{\text {ak }}$, J. Ebr $^{\mathrm{y}}$, R. Engel ${ }^{\text {ah }}$, M. Erdmann ${ }^{\text {al }}$, C.O. Escobar ${ }^{\mathrm{p}}$, A. Etchegoyen ${ }^{\mathrm{b}}$, P. Facal San Luis ${ }^{\mathrm{ci}}$,

I. Fajardo Tapia ${ }^{\text {be }}$, H. Falcke ${ }^{\text {bf,bi }}$, G. Farrar ${ }^{c d}$, A.C. Fauth ${ }^{\text {p }}$, N. Fazzini ${ }^{\text {ca }}$, A.P. Ferguson ${ }^{\text {bw }}$, A. Ferrero ${ }^{b}$, B. Fick $^{c c}$, A. Filevich ${ }^{\text {b }}$, A. Filipčič ${ }^{\text {bm,bn }}$, S. Fliescher ${ }^{\text {al }}$, C.E. Fracchiolla ${ }^{\text {by }}$, E.D. Fraenkel ${ }^{\text {bg }}$, U. Fröhlich $^{\text {an }}$, B. Fuchs ${ }^{\text {a }}$, R. Gaior ${ }^{\text {ad }}$, R.F. Gamarra ${ }^{\text {b }, ~ S . ~ G a m b e t t a ~}{ }^{\text {ao }}$, B. García ${ }^{\text {, }}$ D. García Gámez ${ }^{\text {br }}$, D. Garcia-Pinto bp , A. Gascon ${ }^{\text {br }}$, H. Gemmeke ${ }^{\text {ai }}$, K. Gesterling ${ }^{\text {ck }}$, P.L. Ghia ${ }^{\text {ad,ay }}$, U. Giaccari ${ }^{\text {ar }}$, M. Giller ${ }^{\text {bk }}$, H. Glass ${ }^{\text {ca }}$, M.S. Gold ${ }^{\text {ck }}$, G. Golup ${ }^{\text {a }}$, F. Gomez Albarracin ${ }^{\text {e }}$, M. Gómez Berisso ${ }^{\text {a }}$, P. Gonçalves ${ }^{\text {bl }}$, D. Gonzalez ${ }^{\text {ak }}$, J.G. Gonzalez ${ }^{\text {ak }}$, B. Gookin ${ }^{\text {by }}$, D. Góra ${ }^{\text {aj,bj }}$, A. Gorgi ${ }^{\text {ay }}$, P. Gouffon ${ }^{\circ}$, S.R. Gozzini ${ }^{\text {bu }}$, E. Grashorn ${ }^{\text {cf }}$, S. Grebe ${ }^{\text {bf }}$, N. Griffith ${ }^{\text {cf }}$, M. Grigat ${ }^{\text {al }}$, A.F. Grillo $^{\text {az }}$, Y. Guardincerri ${ }^{\text {d }}$, F. Guarino as , G.P. Guedes ${ }^{\mathrm{q}}$, A. Guzman ${ }^{\text {be }}$, J.D. Hague ${ }^{\text {ck }}$, P. Hansen ${ }^{\mathrm{e}}$, D. Harari $^{\text {a }}$, S. Harmsma ${ }^{\text {bg,bh }}$, J.L. Harton ${ }^{\text {by }}$, A. Haungs ${ }^{\text {ah }}$, T. Hebbeker ${ }^{\text {al }}$, D. Heck ${ }^{\text {ah }}$, A.E. Herve ${ }^{k}$, C. Hojvat $^{\text {ca }}$, N. Hollon ${ }^{\text {ci }}$, V.C. Holmes ${ }^{k}$, P. Homola ${ }^{\text {bj }}$, J.R. Hörandel ${ }^{\text {bf }}$, A. Horneffer ${ }^{\text {bf }}$, M. Hrabovský ${ }^{\mathrm{y}, z}$, T. Huege ${ }^{\text {ah }}$, A. Insolia ${ }^{\text {au }}$, F. Ionita ${ }^{\text {ci }}$, A. Italiano ${ }^{\text {au }}$, C. Jarne ${ }^{\mathrm{e}}$, S. Jiraskova ${ }^{\text {bf }}$, K. Kadija ${ }^{\text {w }}$, K.H. Kampert ${ }^{\text {ag }}$, P. Karhan ${ }^{\mathrm{x}}$, P. Kasper ${ }^{\text {ca }}$, B. Kégl ${ }^{\text {ac }}$, B. Keilhauer ${ }^{\text {ah }}$, A. Keivani ${ }^{c b}$, J.L. Kelley ${ }^{\text {bf }}$, E. Kemp ${ }^{\text {p }}$, R.M. Kieckhafer ${ }^{\text {cc }}$, H.O. Klages ${ }^{\text {ah }}$, M. Kleifges ${ }^{\text {ai }}$, J. Kleinfeller ${ }^{\text {ah }}$, J. Knapp ${ }^{\text {bu }}$, D.-H. Koang ${ }^{\text {ae }}$, K. Kotera ${ }^{\text {ci }}$, N. Krohm ${ }^{\text {ag }}$, O. Krömer ${ }^{\text {ai }}$,

D. Kruppke-Hansen $^{\text {ag }}$, F. Kuehn ${ }^{\text {ca }}$, D. Kuempel ${ }^{\text {ag }}$, J.K. Kulbartz ${ }^{a m}$, N. Kunka ${ }^{a i}$, G. La Rosa ${ }^{a x}$, C. Lachaud $^{\text {ab }}$, P. Lautridou ${ }^{\text {af }}$, M.S.A.B. Leão ${ }^{t}$, D. Lebrun ${ }^{\text {ae }}$, P. Lebrun ${ }^{\text {ca }}$, M.A. Leigui de Oliveira ${ }^{t}$, A. Lemiere ${ }^{\text {aa }}$, A. Letessier-Selvon ${ }^{\mathrm{ad}}$, I. Lhenry-Yvon ${ }^{\mathrm{aa}}$, K. Link ${ }^{\mathrm{ak}}$, R. López ${ }^{\mathrm{bb}}$, A. Lopez Agüera ${ }^{\mathrm{bs}}, \mathrm{K}_{\text {. Louedec }}{ }^{\mathrm{ac}}$, J. Lozano Bahilo $^{\text {br }}$, A. Lucero ${ }^{\text {bay }}$, M. Ludwig ${ }^{\text {ak }}$, H. Lyberis $^{\text {aa }}$, M.C. Maccarone ${ }^{\text {ax }}$, C. Macolino ${ }^{\text {ad }}$, S. Maldera ${ }^{\text {ay }}$, D. Mandat ${ }^{\mathrm{y}}$, P. Mantsch ${ }^{\text {ca }}$, A.G. Mariazzi ${ }^{\mathrm{e}}$, J. Marin ${ }^{\mathrm{i}, a y}$, V. Marin ${ }^{\text {af }}$, I.C. Maris ${ }^{\text {ad }}$, H.R. Marquez Falcon ${ }^{\text {bd }}$, G. Marsella ${ }^{\text {aw }}$, D. Martello ${ }^{\text {ar }}$, L. Martin ${ }^{\text {af }}$, H. Martinez ${ }^{\text {bc }}$, O. Martínez Bravo ${ }^{\text {bb }}$, H.J. Mathes ${ }^{\text {ah }}$, J. Matthews $^{\text {cb,ch }}$, J.A.J. Matthews ${ }^{\text {ck }}$, G. Matthiae ${ }^{\text {at }}$, D. Maurizio ${ }^{\text {av }}$, P.O. Mazur ${ }^{\text {ca }}$, G. Medina-Tanco ${ }^{\text {be }}$, M. Melissas ${ }^{\text {ak }}$, D. Melo ${ }^{\text {bav }}$, E. Menichetti ${ }^{\text {av }}$, A. Menshikov ${ }^{\text {ai }}$, P. Mertsch ${ }^{\text {bt }}$, C. Meurer ${ }^{\text {al }}$, S. Mićanović w M.I. Micheletti ${ }^{g}$, W. Miller ${ }^{\text {ck }}$, L. Miramonti ${ }^{\text {aq }}$, S. Mollerach ${ }^{\mathrm{a}}$, M. Monasor ${ }^{\mathrm{ci}}$, D. Monnier Ragaigne ${ }^{\mathrm{ac}}$, 
F. Montanet ${ }^{\mathrm{ae}}$, B. Morales ${ }^{\text {be }}$, C. Morello ${ }^{\mathrm{ay}}$, E. Moreno ${ }^{\mathrm{bb}}$, J.C. Moreno $^{\mathrm{e}}$, C. Morris ${ }^{\mathrm{cf}}$, M. Mostafá ${ }^{\text {by }}$, C.A. Moura ${ }^{\text {t,as }}$, S. Mueller ${ }^{\text {ah }}$, M.A. Muller ${ }^{p}$, G. Müller $^{\text {al }}$, M. Münchmeyer ${ }^{\text {ad }}$, R. Mussa ${ }^{\text {av }}$, G. Navarra ${ }^{\text {ay,1, }}$, J.L. Navarro ${ }^{\text {br }}$, S. Navas ${ }^{\text {br }}$, P. Necesal ${ }^{\mathrm{y}}$, L. Nellen ${ }^{\text {be }}$, A. Nelles ${ }^{\text {bf }}$, P.T. Nhung ${ }^{\text {cn }}$, L. Niemietz ${ }^{\text {ag }}$,

N. Nierstenhoefer ${ }^{\mathrm{ag}}, \mathrm{D}$. Nitz ${ }^{\mathrm{cc}}, \mathrm{D}$. Nosek $^{\mathrm{x}}$, L. Nožka ${ }^{\mathrm{y}}$, M. Nyklicek $^{\mathrm{y}}$, J. Oehlschläger $^{\mathrm{ah}}$, A. Olinto ${ }^{\text {ci }}$, P. Oliva $^{\text {ag }}$, V.M. Olmos-Gilbaja ${ }^{\text {bs }}$, M. Ortiz ${ }^{\text {bp }}$, N. Pacheco ${ }^{\text {bq }}$, D. Pakk Selmi-Dei ${ }^{\text {p }}$, M. Palatka ${ }^{\mathrm{y}}$, J. Pallotta ${ }^{\mathrm{c}}$, N. Palmieri $^{\text {ak }}$, G. Parente $^{\text {bs }}$, E. Parizot ${ }^{\text {ab }}$, A. Parra ${ }^{\text {bs }}$, R.D. Parsons ${ }^{\text {bu }}$, S. Pastor ${ }^{\text {bo }}$, T. Paul ${ }^{\text {ce }}$, M. Pech $^{\mathrm{y}}$, J. Pȩkala ${ }^{\text {bj }}$, R. Pelayo ${ }^{\text {bs }}$, I.M. Pepe ${ }^{\mathrm{s}}$, L. Perrone ${ }^{\mathrm{aw}}$, R. Pesce $^{\text {ao }}$, E. Petermann $^{\mathrm{cj}}$, S. Petrera ${ }^{\text {ap }}$, P. Petrinca ${ }^{\text {at }}$, A. Petrolini ${ }^{\text {ao }}$, Y. Petrov ${ }^{\text {by }}$, J. Petrovic ${ }^{\text {bh }}$, C. Pfendner ${ }^{c l}$, N. Phan ${ }^{\text {ck }}$, R. Piegaia ${ }^{\text {d }}$, T. Pierog ${ }^{\text {ah }}$, P. Pieroni ${ }^{\text {d }}$, M. Pimenta ${ }^{\text {bl }}$, V. Pirronello ${ }^{\text {au }}$, M. Platino ${ }^{\text {b }}$, V.H. Ponce ${ }^{\mathrm{a}}$, M. Pontz ${ }^{\text {an }}$, P. Privitera ${ }^{\text {ci }}$, M. Prouza ${ }^{\mathrm{y}}$, E.J. Quel ${ }^{\mathrm{c}}$, S. Querchfeld ${ }^{\mathrm{ag}}{ }$ J. Rautenberg $^{\text {ag }}$, O. Ravel ${ }^{\text {af }}$, D. Ravignani ${ }^{\text {b }}$, B. Revenu ${ }^{\text {af }}$, J. Ridky ${ }^{\mathrm{y}}$, S. Riggi ${ }^{\text {au,bs }}$, M. Risse ${ }^{\text {an }}$, P. Ristori ${ }^{c}$, H. Rivera $^{\text {aq }}$, V. Rizi ${ }^{\text {ap }}$, J. Roberts $^{\text {cd }}$, C. Robledo ${ }^{\text {bb }}$, W. Rodrigues de Carvalho ${ }^{\text {o,bs }}$, G. Rodriguez $^{\text {bs }}$, J. Rodriguez Martino ${ }^{\text {i,au }}$, J. Rodriguez Rojo ${ }^{\text {i }}$, I. Rodriguez-Cabo ${ }^{\text {bs }}$, M.D. Rodríguez-Frías ${ }^{\text {bq }}$, G. Ros ${ }^{\text {bq }}$, J. Rosado ${ }^{\text {bp }}$, T. Rossler ${ }^{\mathrm{z}}$, M. Roth ${ }^{\text {ah }}$, B. Rouillé-d'Orfeuil ${ }^{\text {ci }}$, E. Roulet ${ }^{a}$, A.C. Rovero ${ }^{f}$, C. Rühle ${ }^{\text {ai }}$, F. Salamida ${ }^{\text {ah,ap }}$, H. Salazar ${ }^{\text {bb }}$, G. Salina ${ }^{\text {at }}$, F. Sánchez ${ }^{\text {b }}$, M. Santander ${ }^{\text {i }}$, C.E. Santo ${ }^{\text {bl }}$, E. Santos ${ }^{\text {bl }}$, E.M. Santos ${ }^{\text {u }}$, F. Sarazin ${ }^{\text {bx }}$, B. Sarkar ag, S. Sarkar ${ }^{\text {bt }}$, R. Sato ${ }^{i}$, N. Scharf ${ }^{\text {al }}$, V. Scherini ${ }^{\text {aq }}$, H. Schieler ${ }^{\text {ah }}$, P. Schiffer ${ }^{\text {al }}$, A. Schmidt ${ }^{\text {ai }}$, F. Schmidt ${ }^{\text {ci }}$, T. Schmidt ${ }^{\text {ak }}$, O. Scholten ${ }^{\text {bg }}$, H. Schoorlemmer ${ }^{\text {bf }}$, J. Schovancova ${ }^{\mathrm{y}}$, P. Schovánek ${ }^{\mathrm{y}}$, F. Schröder ${ }^{\text {ah }}$, S. Schulte ${ }^{\text {al }}$,

D. Schuster ${ }^{\text {bx }}$, S.J. Sciutto ${ }^{\mathrm{e}}$, M. Scuderi ${ }^{\text {au }}$, A. Segreto ${ }^{a x}$, M. Settimo ${ }^{\text {an }}$, A. Shadkam ${ }^{c b}$, R.C. Shellard ${ }^{1, m}$, I. Sidelnik $^{\text {b }}$, G. Sigl ${ }^{\text {am }}$, H.H. Silva Lopez ${ }^{\text {be }}, A$. Śmiałkowski ${ }^{\text {bk }}$, R. Šmída $^{\text {y,ah }}{ }^{\text {, G.R. Snow }}{ }^{\text {cj }}$, P. Sommers ${ }^{\text {cg }}$, J. Sorokin ${ }^{\text {k }}$, H. Spinka ${ }^{\text {bv,ca }}$, R. Squartini ${ }^{i}$, J. Stapleton ${ }^{\text {cf }}$, J. Stasielak ${ }^{\text {bj }}$, M. Stephan ${ }^{\text {al }}$, E. Strazzeri ${ }^{\text {ax }}$, A. Stutz $^{\text {ae }}$, F. Suarez ${ }^{\text {b }}$, T. Suomijärvi ${ }^{\text {aa }}$, A.D. Supanitsky ${ }^{\text {f,be }}$, T. Šuša ${ }^{\text {w }}$, M.S. Sutherland ${ }^{\text {cb,cf }}$, J. Swain ${ }^{\text {ce }}$, Z. Szadkowski ${ }^{\text {ag,bk }}$, M. Szuba ${ }^{\text {ah }}$, A. Tamashiro ${ }^{f}$, A. Tapia ${ }^{b}$, M. Tartare ${ }^{\text {ae }}$, O. Taşcău ${ }^{\text {ag }}$, C.G. Tavera Ruiz ${ }^{\text {be }}$, R. Tcaciuc ${ }^{\text {an }}$, D. Tegolo ${ }^{\text {au,ba }}$, N.T. Thao ${ }^{\text {cn }}$, D. Thomas ${ }^{\text {by }}$, J. Tiffenberg ${ }^{d}$, C. Timmermans ${ }^{\text {bf,bh }}$, D.K. Tiwari ${ }^{\text {bd }}$, W. Tkaczyk ${ }^{\text {bk }}$, C.J. Todero Peixoto ${ }^{n, t}$, B. Tomé ${ }^{\text {bl }}$, A. Tonachini ${ }^{\text {av }}$, P. Travnicek ${ }^{\mathrm{y}}$, D.B. Tridapalli ${ }^{\mathrm{o}}$, G. Tristram $^{\mathrm{ab}}$, E. Trovato $^{\mathrm{au}}$, M. Tueros ${ }^{\text {d,bs }}$, R. Ulrich ${ }^{\text {ah,cg }}$, M. Unger ${ }^{\text {ah }}$, M. Urban ${ }^{\text {ac }}$, J.F. Valdés Galicia ${ }^{\text {be }}$, I. Valiño ${ }^{\text {ah,bs }}$, L. Valore ${ }^{\text {as }}$, A.M. van den Berg ${ }^{\text {bg }}$, E. Varela ${ }^{\text {bb }}$, B. Vargas Cárdenas ${ }^{\text {be }}$, J.R. Vázquez ${ }^{\text {bp }}$, R.A. Vázquez ${ }^{\text {bs }}$, D. Veberič ${ }^{\text {bm,bn }}$, V. Verzi $^{\text {at }}$, J. Vicha ${ }^{\mathrm{y}}$, M. Videla ${ }^{\mathrm{h}}$, L. Villaseñor ${ }^{\text {bd }}$, H. Wahlberg ${ }^{\mathrm{e}}$, P. Wahrlich ${ }^{\mathrm{k}}$, O. Wainberg ${ }^{\mathrm{b}}$, D. Warner ${ }^{\text {by }}$, A.A. Watson ${ }^{\text {bu }}$, M. Weber ${ }^{\text {ai }}$, K. Weidenhaupt ${ }^{\text {al }}$, A. Weindl ${ }^{\text {ah }}$, S. Westerhoff ${ }^{c l}$, B.J. Whelan ${ }^{\mathrm{k}}$, G. Wieczorek ${ }^{\text {bk, }}$, L. Wiencke $^{\text {bx }}$, B. Wilczyńska ${ }^{\text {bj }}$, H. Wilczyński ${ }^{\text {bj }}$, M. Will ${ }^{\text {ah }}$, C. Williams ${ }^{c i}$, T. Winchen $^{\text {al }}$, L. Winders ${ }^{\mathrm{cm}}$, M.G. Winnick ${ }^{\mathrm{k}}$, M. Wommer ${ }^{\text {ah }}$, B. Wundheiler ${ }^{\mathrm{b}}$, T. Yamamoto $^{\text {ci,co, T. Yapici }}{ }^{\mathrm{cc}}$, P. Younk ${ }^{\text {an }}$, G. Yuan $^{\text {cb', }}$ A. Yushkov ${ }^{\text {as,bs }}$, B. Zamorano ${ }^{\text {br }}$, E. Zas ${ }^{\text {bs }}$, D. Zavrtanik ${ }^{\text {bm,bn }}$, M. Zavrtanik ${ }^{\text {bm,bn }}$, I. Zaw ${ }^{\text {cd }}$, A. Zepeda ${ }^{\text {bc }}$, M. Ziolkowski an

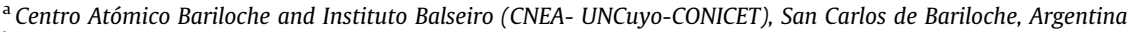

${ }^{\mathrm{b}}$ Centro Atómico Constituyentes (Comisión Nacional de Energía Atómica/CONICET/UTN-FRBA), Buenos Aires, Argentina

${ }^{c}$ Centro de Investigaciones en Láseres y Aplicaciones, CITEFA and CONICET, Argentina

${ }^{\mathrm{d}}$ Departamento de Física, FCEyN, Universidad de Buenos Aires y CONICET, Argentina

e IFLP, Universidad Nacional de La Plata and CONICET, La Plata, Argentina

${ }^{\mathrm{f}}$ Instituto de Astronomía y Física del Espacio (CONICET- UBA), Buenos Aires, Argentina

${ }^{\mathrm{g}}$ Instituto de Física de Rosario (IFIR) - CONICET/U.N.R. and Facultad de Ciencias Bioquímicas y Farmacéuticas U.N.R., Rosario, Argentina

${ }^{\mathrm{h}}$ National Technological University, Faculty Mendoza (CONICET/CNEA), Mendoza, Argentina

i Pierre Auger Southern Observatory, Malargüe, Argentina

j Pierre Auger Southern Observatory and Comisión Nacional de Energía Atómica, Malargüe, Argentina

k University of Adelaide, Adelaide, SA, Australia

${ }^{1}$ Centro Brasileiro de Pesquisas Fisicas, Rio de Janeiro, RJ, Brazil

${ }^{\mathrm{m}}$ Pontifícia Universidade Católica, Rio de Janeiro, RJ, Brazil

${ }^{\mathrm{n}}$ Universidade de São Paulo, Instituto de Física, São Carlos, SP, Brazil

${ }^{\circ}$ Universidade de São Paulo, Instituto de Física, São Paulo, SP, Brazil

${ }^{\mathrm{p}}$ Universidade Estadual de Campinas, IFGW, Campinas, SP, Brazil

${ }^{\mathrm{q}}$ Universidade Estadual de Feira de Santana, Brazil

${ }^{\mathrm{r}}$ Universidade Estadual do Sudoeste da Bahia, Vitoria da Conquista, BA, Brazil

s Universidade Federal da Bahia, Salvador, BA, Brazil

${ }^{\mathrm{t}}$ Universidade Federal do ABC, Santo André, SP, Brazil

${ }^{\mathrm{u}}$ Universidade Federal do Rio de Janeiro, Instituto de Física, Rio de Janeiro, RJ, Brazil

${ }^{\mathrm{v}}$ Universidade Federal Fluminense, EEIMVR, Volta Redonda, RJ, Brazil

${ }^{\mathrm{w}}$ Rudjer Bošković Institute, 10000 Zagreb, Croatia

${ }^{x}$ Charles University, Faculty of Mathematics and Physics, Institute of Particle and Nuclear Physics, Prague, Czech Republic

${ }^{\mathrm{y}}$ Institute of Physics of the Academy of Sciences of the Czech Republic, Prague, Czech Republic

${ }^{z}$ Palacky University, RCATM, Olomouc, Czech Republic

aa Institut de Physique Nucléaire d'Orsay (IPNO), Université Paris 11, CNRS-IN2P3 Orsay, France

${ }^{\mathrm{ab}}$ Laboratoire AstroParticule et Cosmologie (APC), Université Paris 7, CNRS-IN2P3 Paris, France

${ }^{a c}$ Laboratoire de l'Accélérateur Linéaire (LAL), Université Paris 11, CNRS-IN2P3 Orsay, France

${ }^{a d}$ Laboratoire de Physique Nucléaire et de Hautes Energies (LPNHE), Universités Paris 6 et Paris 7, CNRS-IN2P3 Paris, France

ae Laboratoire de Physique Subatomique et de Cosmologie (LPSC), Université Joseph Fourier, INPG, CNRS-IN2P3 Grenoble, France

af SUBATECH, CNRS-IN2P3, Nantes, France
} 
ag Bergische Universität Wuppertal, Wuppertal, Germany

${ }^{\text {ah }}$ Karlsruhe Institute of Technology - Campus North - Institut für Kernphysik, Karlsruhe, Germany

ai Karlsruhe Institute of Technology - Campus North - Institut für Prozessdatenverarbeitung und Elektronik, Karlsruhe, Germany

aj Karlsruhe Institute of Technology - Campus South - Institut für Experimentelle Kernphysik (IEKP), Karlsruhe, Germany

${ }^{\mathrm{ak}}$ Max-Planck-Institut für Radioastronomie, Bonn, Germany

${ }^{\text {al }}$ RWTH Aachen University, III. Physikalisches Institut A, Aachen, Germany

am Universität Hamburg, Hamburg, Germany

an Universität Siegen, Siegen, Germany

${ }^{\text {ao }}$ Dipartimento di Fisica dell'Università and INFN, Genova, Italy

${ }^{\text {ap } U n i v e r s i t a ̀ ~ d e l l ' A q u i l a ~ a n d ~ I N F N, ~ L ' A q u i l a, ~ I t a l y ~}$

${ }^{\mathrm{aq}}$ Università di Milano and Sezione INFN, Milan, Italy

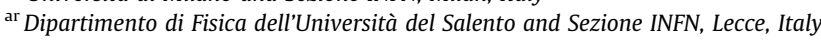

as Università di Napoli "Federico II" and Sezione INFN, Napoli, Italy

at Università di Roma II "Tor Vergata" and Sezione INFN, Roma, Italy

au Università di Catania and Sezione INFN, Catania, Italy

${ }^{\text {av } U n i v e r s i t a ̀ ~ d i ~ T o r i n o ~ a n d ~ S e z i o n e ~ I N F N, ~ T o r i n o, ~ I t a l y ~}$

${ }^{\mathrm{aw}}$ Dipartimento di Ingegneria dell'Innovazione dell'Università del Salento and Sezione INFN, Lecce, Italy

ax Istituto di Astrofisica Spaziale e Fisica Cosmica di Palermo (INAF), Palermo, Italy

ay Istituto di Fisica dello Spazio Interplanetario (INAF), Università di Torino and Sezione INFN, Torino, Italy

az INFN, Laboratori Nazionali del Gran Sasso, Assergi (L'Aquila), Italy

ba Università di Palermo and Sezione INFN, Catania, Italy

${ }^{\mathrm{b}}$ Benemérita Universidad Autónoma de Puebla, Puebla, Mexico

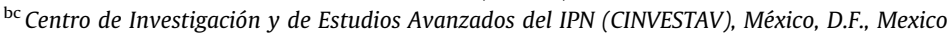

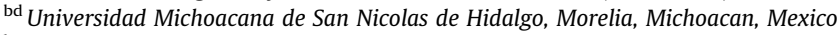

be Universidad Nacional Autonoma de Mexico, Mexico, D.F., Mexico

bf IMAPP, Radboud University, Nijmegen, Netherlands

${ }^{\mathrm{bg}}$ Kernfysisch Versneller Instituut, University of Groningen, Groningen, Netherlands

bh NIKHEF, Amsterdam, Netherlands

${ }^{\text {bi }}$ ASTRON, Dwingeloo, Netherlands

${ }^{\mathrm{bj}}$ Institute of Nuclear Physics PAN, Krakow, Poland

${ }^{\mathrm{bk}}$ University of Łódź, Łódź, Poland

${ }^{\mathrm{bl}}$ LIP and Instituto Superior Técnico, Lisboa, Portugal

${ }^{\mathrm{bm}} J$. Stefan Institute, Ljubljana, Slovenia

bn Laboratory for Astroparticle Physics, University of Nova Gorica, Slovenia

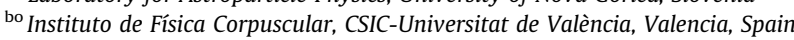

bp Universidad Complutense de Madrid, Madrid, Spain

${ }^{\mathrm{bq}}$ Universidad de Alcalá, Alcalá de Henares (Madrid), Spain

${ }^{\mathrm{br}}$ Universidad de Granada \& C.A.F.P.E., Granada, Spain

bs Universidad de Santiago de Compostela, Spain

${ }^{\mathrm{bt}}$ Rudolf Peierls Centre for Theoretical Physics, University of Oxford, Oxford, United Kingdom

bu School of Physics and Astronomy, University of Leeds, United Kingdom

${ }^{\text {bv }}$ Argonne National Laboratory, Argonne, IL, USA

${ }^{b w}$ Case Western Reserve University, Cleveland, $\mathrm{OH}$, USA

${ }^{b x}$ Colorado School of Mines, Golden, CO, USA

by Colorado State University, Fort Collins, CO, USA

${ }^{\mathrm{bz}}$ Colorado State University, Pueblo, CO, USA

ca Fermilab, Batavia, IL, USA

${ }^{\mathrm{cb}}$ Louisiana State University, Baton Rouge, LA, USA

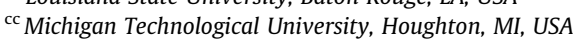

${ }^{\mathrm{cd}}$ New York University, New York, NY, USA

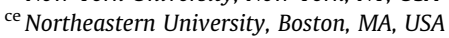

cf Ohio State University, Columbus, OH, USA

cg Pennsylvania State University, University Park, PA, USA

${ }^{\mathrm{ch}}$ Southern University, Baton Rouge, LA, USA

${ }^{c i}$ University of Chicago, Enrico Fermi Institute, Chicago, IL, USA

${ }^{c j}$ University of Nebraska, Lincoln, NE, USA

${ }^{\mathrm{ck}}$ University of New Mexico, Albuquerque, NM, USA

${ }^{\mathrm{cl}}$ University of Wisconsin, Madison, WI, USA

${ }^{\mathrm{cm}}$ University of Wisconsin, Milwaukee, WI, USA

${ }^{\mathrm{cn}}$ Institute for Nuclear Science and Technology (INST), Hanoi, Vietnam

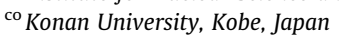

\section{A R T I C L E I N F O}

\section{Article history}

Received 17 June 2011

Received in revised form 23 July 2011

Accepted 1 August 2011

Available online 11 August 2011

Keywords:

Ultra-High Energy Cosmic Rays

Pierre Auger Observatory

\section{A B S T R A C T}

In this paper we introduce the concept of Lateral Trigger Probability (LTP) function, i.e., the probability for an Extensive Air Shower (EAS) to trigger an individual detector of a ground based array as a function of distance to the shower axis, taking into account energy, mass and direction of the primary cosmic ray. We apply this concept to the surface array of the Pierre Auger Observatory consisting of a $1.5 \mathrm{~km}$ spaced grid of about 1600 water Cherenkov stations. Using Monte Carlo simulations of ultra-high energy showers the LTP functions are derived for energies in the range between $10^{17}$ and $10^{19} \mathrm{eV}$ and zenith angles up to $65^{\circ}$. A parametrization combining a step function with an exponential is found to reproduce them very well in the considered range of energies and zenith angles. The LTP functions can also be obtained from data

\footnotetext{
1 Deceased

E-mail address: auger_spokespersons@fnal.gov
} 
Extensive Air Showers Trigger performance Surface detector Hybrid detector using events simultaneously observed by the fluorescence and the surface detector of the Pierre Auger Observatory (hybrid events). We validate the Monte Carlo results showing how LTP functions from data are in good agreement with simulations.

(c) 2011 Elsevier B.V. All rights reserved.

\section{Introduction}

The Pierre Auger Observatory has been conceived to study the origin and the nature of ultra high-energy cosmic rays. Because of the scarcity of the flux at the highest energies, their direct measurement from space is technically unfeasible and the use of very large detectors is required at the ground. What can be observed is the extensive air shower of secondary particles produced in the propagation through the atmosphere. The Pierre Auger Observatory is located near Malargüe, Argentina, at $1400 \mathrm{~m}$ a.s.l. and it employes two independent and complementary measurement techniques [1]. The surface array (SD), consisting of about 1600 water Cherenkov detectors on a triangular grid of $1.5 \mathrm{~km}$ spacing covering an area of approximately $3000 \mathrm{~km}^{2}$, records the secondary particles at the ground and thus samples their lateral density distribution. The fluorescence detector (FD), consisting of 24 telescopes at four sites, overlooks the surface array and observes the longitudinal profile of air showers by collecting the fluorescence light emitted along the path through the atmosphere [2]. Unlike the surface detector array with its nearly $100 \%$ duty cycle, the FD can only operate on clear and moonless nights giving an overall duty cycle of about $13 \%$ [3]. As a consequence, only a fraction of showers are observed by both detectors. For these events, called hereafter "hybrid", the combination of information from the surface array and the fluorescence telescopes enhances the reconstruction capability. Energy and direction reconstruction accuracy of hybrid events is in fact better than the one the SD and FD could achieve independently.

One of the main goals of the Pierre Auger Observatory is to measure the flux of cosmic rays at the highest energies. This task relies on an accurate determination of the detector exposure for SD-only [4] and hybrid [3] operation modes. The hybrid exposure is calculated using the simultaneous simulation of FD and SD response. Besides the dependence on energy and distance to an FD-site, the hybrid exposure is influenced by several factors including the atmospheric conditions, the trigger status of all active detectors and their instantaneous data taking configuration. The calculation of the SD response is based on the deep knowledge of the array capability to trigger once a shower with a given energy and zenith angle hits the ground. Since the trigger in an EAS array is always a combination of trigger states of neighboring detectors, the acceptance of any EAS array is directly connected to the probability that an individual detector triggers when a shower lands at a certain distance from it. This defines the concept of "Lateral Trigger Probability" function. This function has been used as a powerful tool for simulations in the analysis for the measurement of the hybrid energy spectrum [5] and of the atmospheric depth at shower maximum [6].

The trigger design of the Auger surface detector is described in detail in [4]. Each water Cherenkov detector of the surface array has a $10 \mathrm{~m}^{2}$ water surface area and $1.2 \mathrm{~m}$ water depth, with three 9 in photomultiplier tubes (PMTs) looking through optical coupling material into the water volume, which is contained in a Tyvek ${ }^{\circledR}$ reflective liner. The signals provided by each PMT are digitised by $40 \mathrm{MHz}$ 10-bit flash analog to digital converters (FADCs) [1]. The achieved dynamical range is sufficient to cover with good precision both the signals produced in the detectors near to the shower axis $(\sim 1000$ particles $/ \mu \mathrm{s})$ and those produced far from the shower axis $(\sim 1$ particle $/ \mu s)$. We recall here the basic structure of the used trigger algorithms. The two first levels (T1 and T2) are formed at each surface detector. Each trigger level can be divided in two modes, a threshold trigger (TH) and a time-over-threshold trigger (ToT). The first level threshold trigger (TH-T1) requires the coincidence of the signals from the three PMTs equipping each station, each PMT signal being above 1.75 "Vertical Equivalent Muon" (VEM). ${ }^{2}$ The TH-T1 trigger is used to reduce the rate due to atmospheric muons to $\sim 100 \mathrm{~Hz}$ and can reach the second level, TH-T2, when the peak signal reaches at least 3.2 VEM in coincidence between 3 PMTs signals, further reducing the rate to $\sim 20 \mathrm{~Hz}$. The second mode, the ToT, requires at least 13 time bins (i.e. more than $325 \mathrm{~ns}$ ) in 120 FADC bins of a sliding window of $3 \mu$ s to be above a threshold of 0.2 VEM in coincidence in 2 out of 3 PMTs. Timeover-threshold trigger stations are automatically promoted to the second level. The threshold trigger is especially efficient at detecting strong narrow signals, mostly encountered in horizontal showers or close to the axis of vertical showers. On the other hand, the ToT is intended to select sequences of small signals spread in time. This is typical of low energy vertical showers dominated by an electromagnetic component or of high energy showers triggering stations at large distance from the shower axis because of muons produced high in the atmosphere.

Higher level triggers are obtained by requiring the spatial and temporal coincidence of at least three stations satisfying the T2 conditions. In particular, for zenith angles below $60^{\circ}$, the full efficiency for SD is reached at $10^{18.5} \mathrm{eV}$ [4]. In addition, if at least one FD telescope triggers in coincidence with one second level trigger station, a hybrid trigger is formed. Since FD has a lower energy threshold, hybrid events are also detected below the minimum energy for an independent SD trigger. For zenith angles below $60^{\circ}$, the hybrid detector reaches nearly full efficiency at $10^{18} \mathrm{eV}$ [3].

In Section 2, the concept of a Lateral Trigger Probability (LTP) function is formalized and applied to the particular case of the surface detector of the Pierre Auger Observatory. In Section 3, the LTP functions for a single time-over-threshold trigger station are derived and parametrized for different primary particles (proton, iron, photon) and their dependence on energy and zenith angle is explored for zenith angles up to $65^{\circ}$ and for energies between $10^{17}$ and $10^{19} \mathrm{eV}$. This energy range is relevant as it covers the interval in which the SD-only and the hybrid detection mode become fully efficient. The dependence on the choice of the hadronic interaction models is also discussed in Section 3. In Section 4, hybrid data are finally used to validate the simulation and to estimate the impact of weather conditions on the observed efficiency. The LTP functions are found to reproduce very well the detector response over a wide range of energies and zenith angles.

\section{Concept of Lateral Trigger Probability}

The trigger probability of a single water Cherenkov detector depends on several independent physical parameters: (i) the characteristics of the primary cosmic ray that initiates an air shower, e.g.,

\footnotetext{
2 The distribution of measured light due to atmospheric muons produces a peak in the PMT charge distribution, $Q_{\mathrm{VEM}}^{\text {peak }}$ (or VEM in short), as well as a peak in that of the pulse height, $I_{\mathrm{VEM}}^{\text {peak }}$, both of them being proportional to those produced by a vertical through-going muon [1]
} 

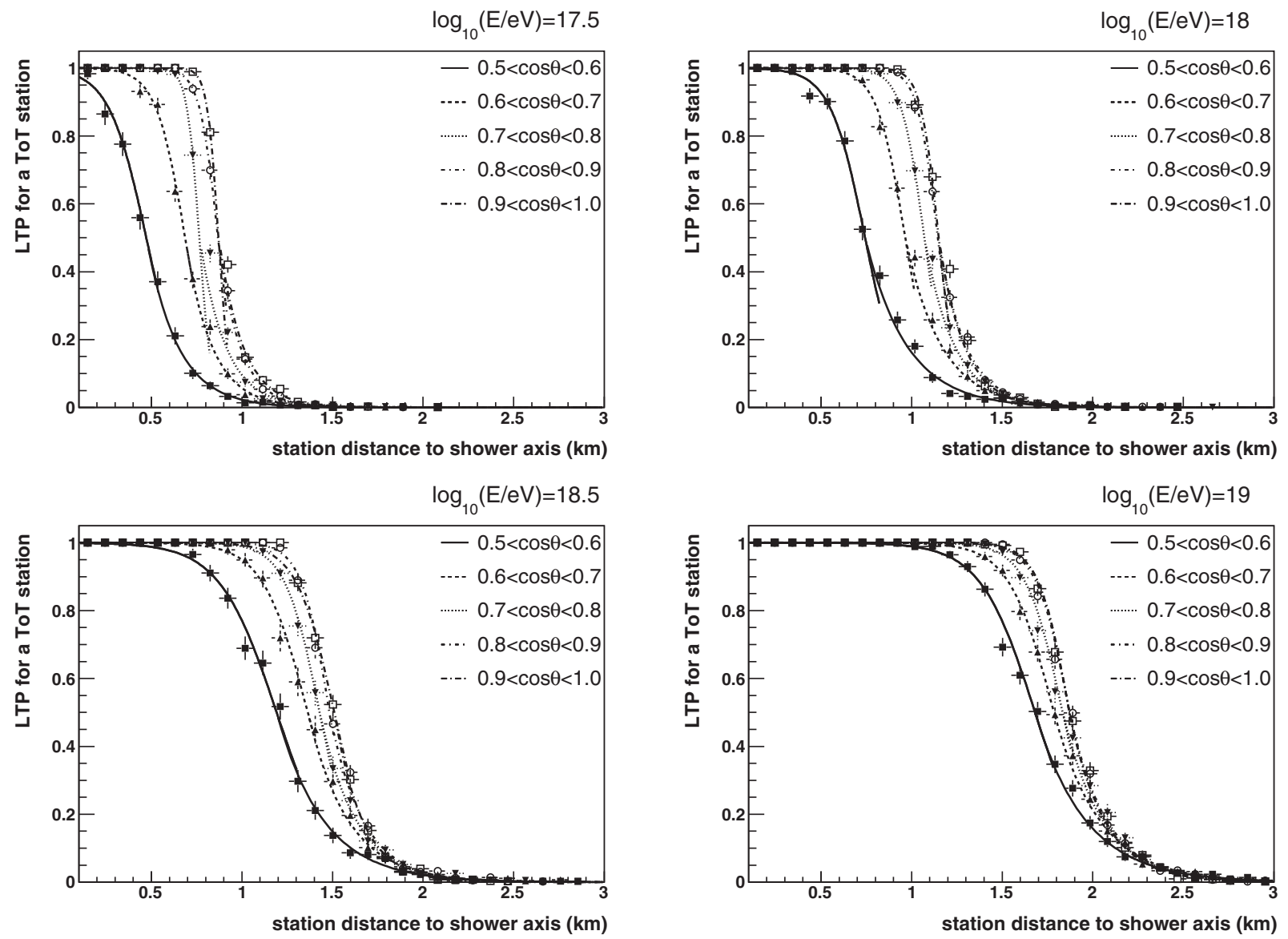

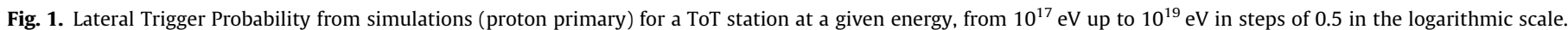
Different bins of $\cos \theta$ are also shown together with a fit performed according to Eq. (2), superimposed as a continuous line.
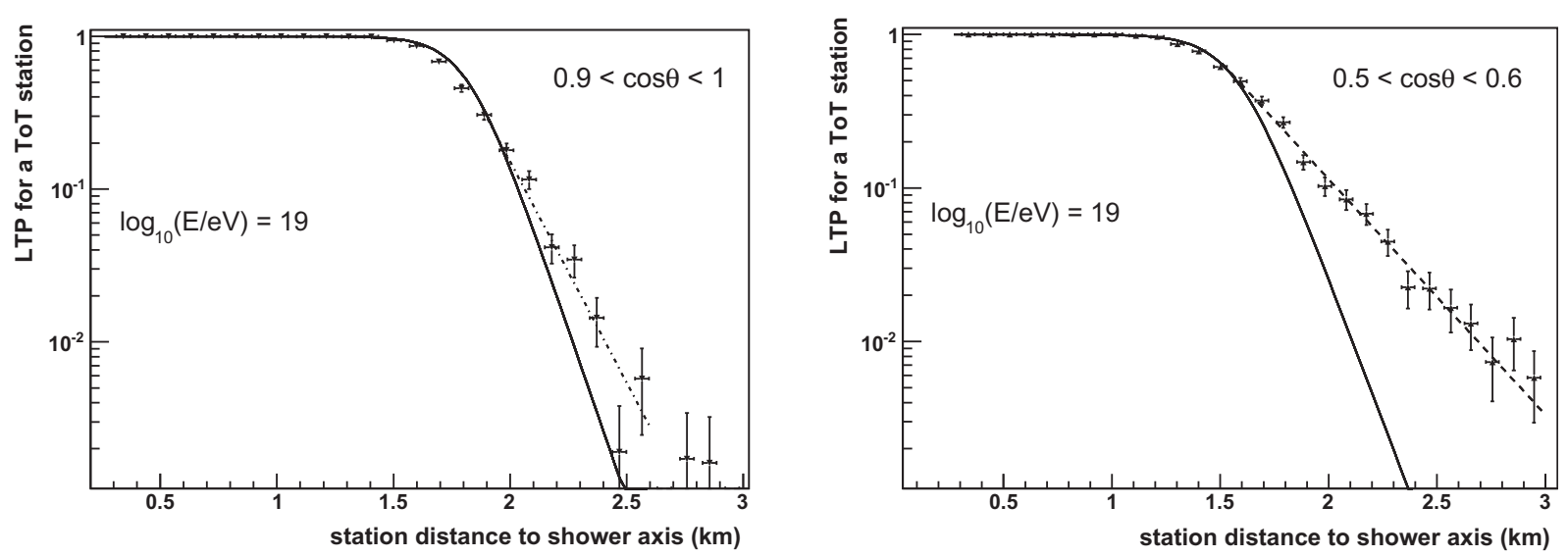

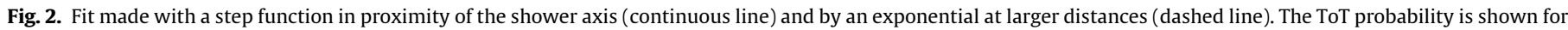
vertical (left) and inclined (right) showers at energy of $10^{19} \mathrm{eV}$.

its energy and mass, (ii) the type and geometry of the detector used to observe air showers (in the following we will only study water Cherenkov detectors used for the surface detector array of the Pierre Auger Observatory), (iii) the trigger condition used to detect a signal from air showers, (iv) the geometry of the incoming shower, e.g. its incidence zenith angle and position with respect to the detector. To formalize these dependencies we define the Lateral Trigger Probability function $\Lambda_{A, E, \theta, T r}(r, \phi)$ as the probability to trigger on an air shower induced by a primary particle of energy
$E$, mass $A$ and zenith angle $\theta$ [7]. Here, $r$ and $\phi$ are the radial coordinates of the single detector in the plane normal to the shower axis (shower frame). Using a trigger condition $T r$, this probability is simply given by:

$\Lambda_{A, E, \theta, T r}(r, \phi)=\frac{N_{1}}{N_{1}+N_{0}}$.

where $N_{1}$ and $N_{0}$ are respectively the number of triggered and untriggered detectors with coordinates $r$ and $\phi$ in the shower frame. 


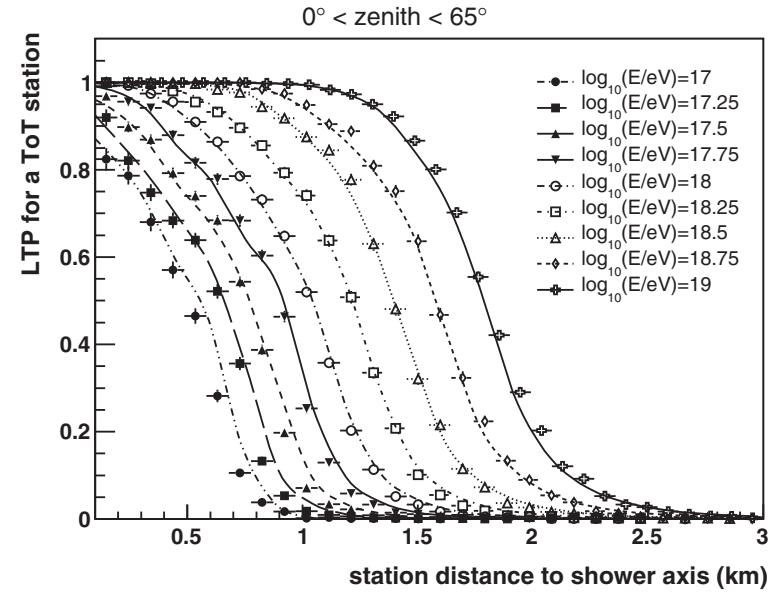

Fig. 3. Lateral Trigger Probability for a ToT station as a function of station distance to shower axis and for different energies (proton primary). The outcome of the parametrization is superimposed as a line. All zenith angles up to $65^{\circ}$ are merged.
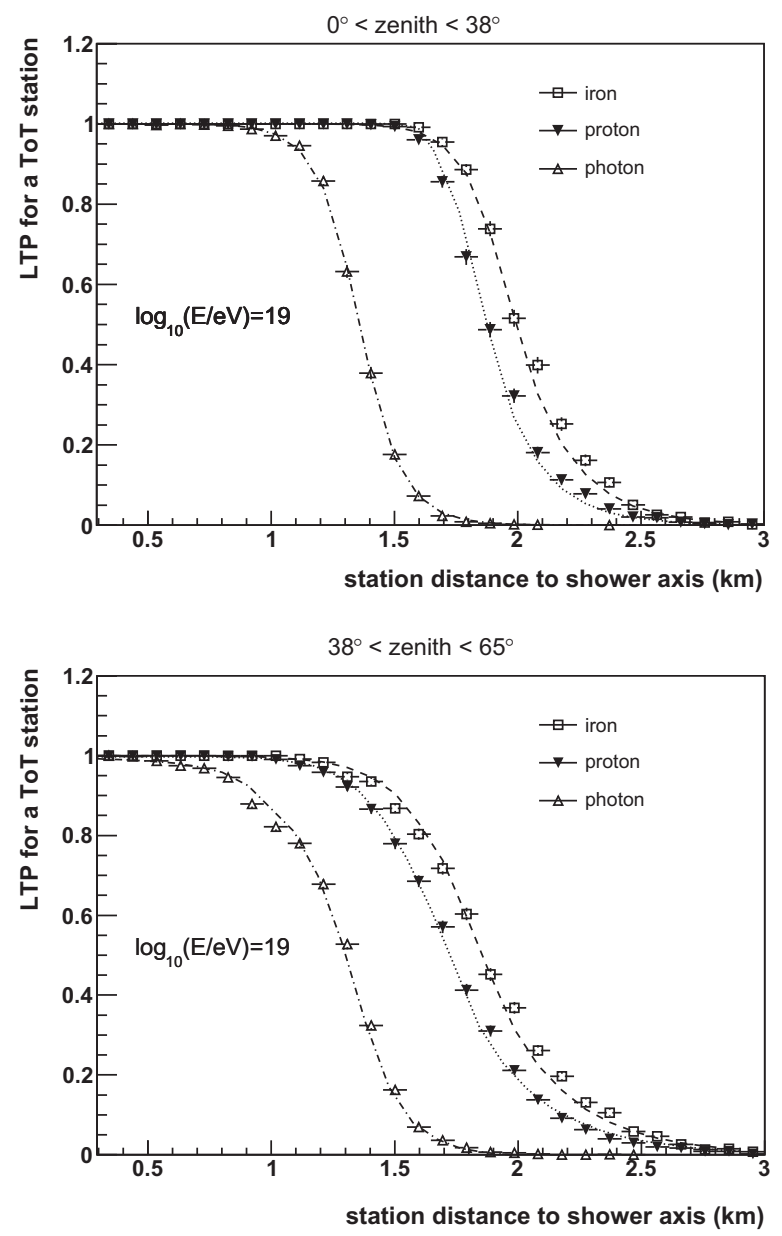

Fig. 4. Lateral Trigger Probability for a ToT station. Proton, iron and photon primaries of energy $10^{19} \mathrm{eV}$ for two zenith angle ranges, $0^{\circ}-38^{\circ}$ (top) and $38^{\circ}-65^{\circ}$ (bottom). The outcome of the parametrization is superimposed as a continuous line.

\section{Simulations}

The LTP functions have been derived using detailed simulations of the EAS development and of the detector response. The simulation sample consists of about 15000 CORSIKA [8] showers (proton,

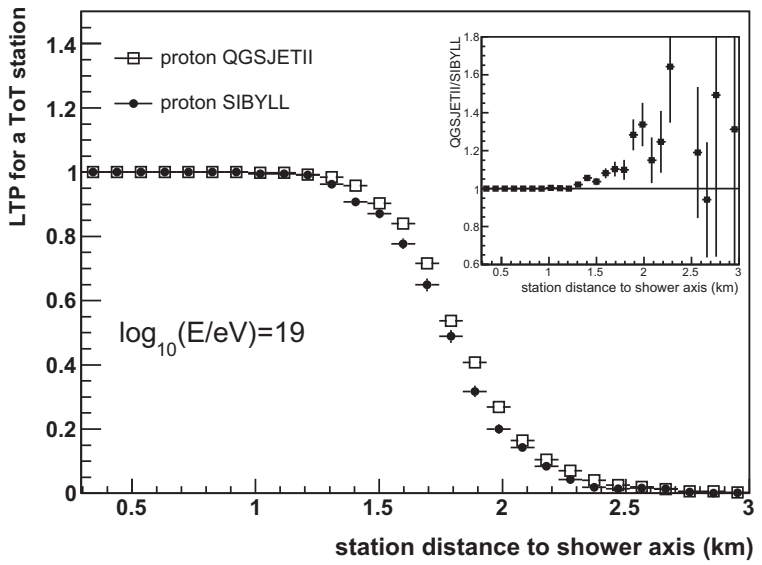

Fig. 5. Lateral Trigger Probability for a ToT station (zenith angle between $0^{\circ}$ and $65^{\circ}$ ). Proton primary at energy of $10^{19} \mathrm{eV}$ with QGSJETII and SIBYLL. The ratio QGSJETII/SIBYLL is shown in the inset.

iron and photon primaries) with zenith angle distributed as $\sin \theta$ $\cos \theta\left(\theta<65^{\circ}\right)$ and energies ranging between $10^{17}$ and $10^{19} \mathrm{eV}$ in steps of 0.25 in the logarithmic scale. A "thin sampling" mechanism at the level of $10^{-6}$ (optimal thinning) is applied following the standard method used for CORSIKA simulation with energies larger than $10^{16} \mathrm{eV}$ [9]. The showers have been generated with the models QGSJETII [10] and FLUKA [11] for high and low energy hadronic interactions.

In the simulation, the position of the shower core (i.e. the intersection of the shower axis with the ground) is uniformly distributed over the surface array and each shower is used 5 times, each time with a different core position, in order to cover different areas of the array and explore all the detector configurations. The surface detector response is simulated using GEANT4 [12] and adopting the sampling procedure to regenerate particles in a ground detector from thinned air shower simulations as described in [13]. The entire detector simulation is carried out within the framework provided by the Auger Offline software [14].

The trigger status of SD stations is inspected within a radius of $3 \mathrm{~km}$ from the shower axis and the Lateral Trigger Probability is then derived according to Eq. (1). At distances larger than $3 \mathrm{~km}$, the trigger efficiency is negligibly small for the class of events studied in this paper. All trigger modes of the surface detector are simulated in detail at all levels. However, for events with zenith angles below $65^{\circ}$, the majority of the stations forming a second level trigger satisfy the ToT condition. In particular, for the considered zenith angles, the fraction of TH-T2 trigger stations not being also ToT is about $1 \%$, approximately independent of the energy. Thus, we focus the analysis on the ToT stations.

The Lateral Trigger Probability for a ToT station is shown in Fig. 1 at a given energy and for different ranges of the cosine of the zenith angle $\theta$. The maximum effective distance for detection increases with energy and, for a given energy, with the cosine of the zenith angle, i.e. events with larger zenith angle tend to trigger less due to the attenuation of their electromagnetic component. For moderately inclined showers, an asymmetry is expected in the signal detected in the stations placed at the same distance to the shower axis but with different azimuth in the shower frame [15]. Indeed, secondary particles arriving earlier traverse less atmosphere and are less attenuated than the late ones. As a consequence, early stations may exhibit larger trigger probabilities and produce larger signals. Actually, for zenith angles below $65^{\circ}$, this effect has been found to have a quite low influence on the trigger probability, only noticeable above $30^{\circ}$ (in simulations as well as in the data). In the following we consider LTP functions averaged 

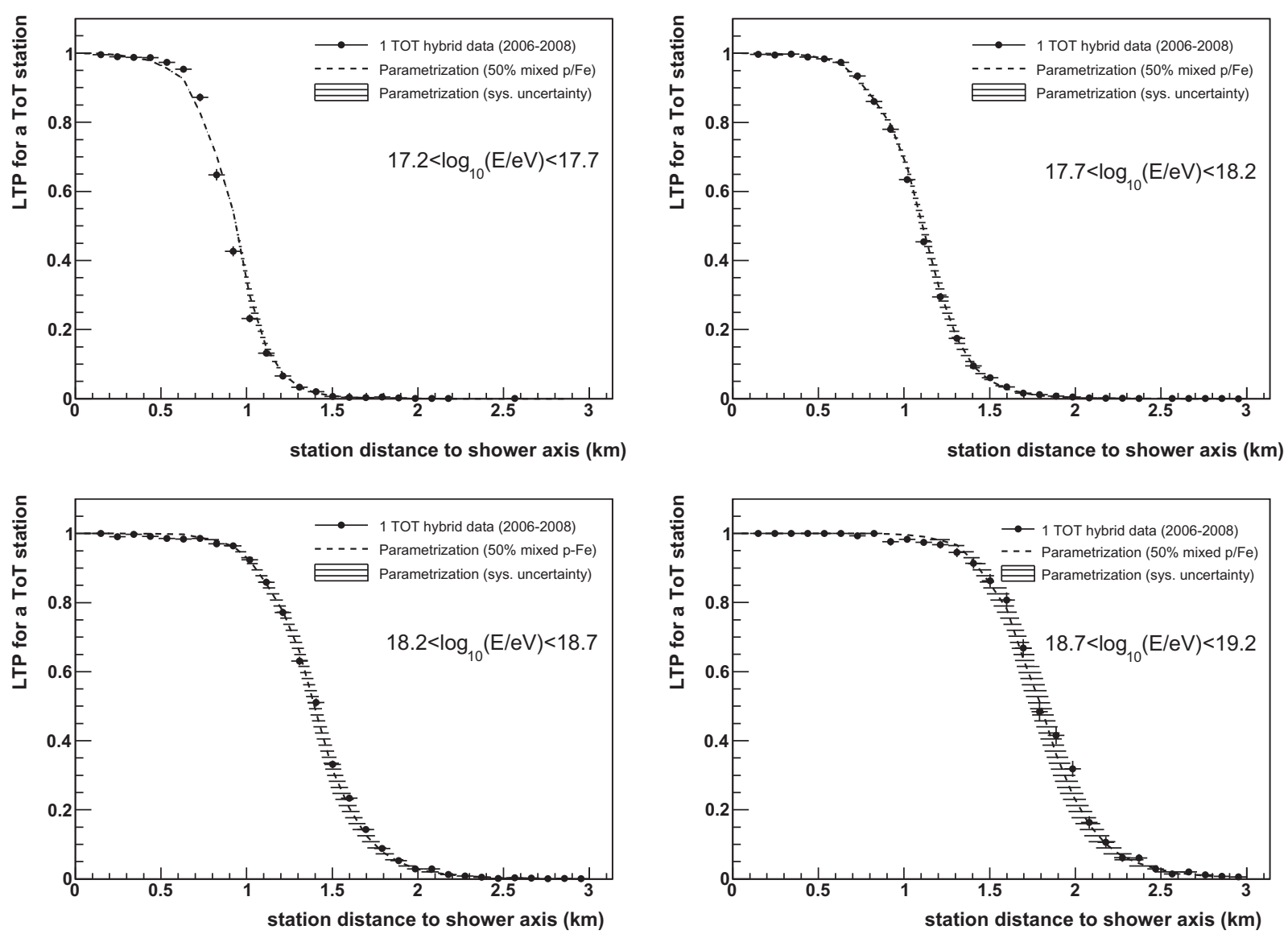

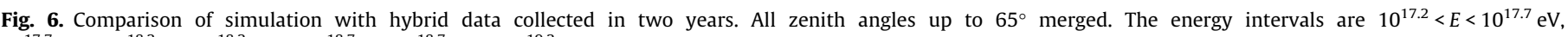
$10^{17.7}<E<10^{18.2} \mathrm{eV}, 10^{18.2}<E<10^{18.7} \mathrm{eV}, 10^{18.7}<E<10^{19.2} \mathrm{eV}$.

over all azimuths in the showers frame. A more detailed treatment including the azimuthal dependence does not introduce measurable differences for acceptance calculations.

A fit combining a step function (close to the axis) with an exponential (further away) reproduces reasonably well the full simulated data set. The form of the fit function used is:

$\operatorname{LTP}(r)= \begin{cases}\frac{1}{1+e^{-\frac{r-R_{0}}{\Delta R}}} & r \leqslant R_{0} \\ \frac{1}{2} e^{C \cdot\left(r-R_{0}\right)} & r>R_{0}\end{cases}$

where $R_{0}, \Delta R$ and $C$ are free fit parameters, with $R_{0}$ being the distance where LTP is equal to 0.5. A fit performed according to Eq. (2) is superimposed on each plot shown in Fig. 1. As an example, the ToT trigger probability at energy $E=10^{19} \mathrm{eV}$ and for two angular bins (vertical showers on the left and showers with larger zenith angle on the right) is shown in Fig. 2: the exponential can reproduce very well the tail of the probability distribution at large distances from axis, in particular for inclined events. The dependences of fit parameters $R_{0}$, $\Delta R$ and $C$ on energy and zenith angle can be parametrized by quadratic polynomials in the variables $\cos \theta$ and $\log _{10}(E / \mathrm{eV})$. The corresponding coefficients are tabulated in the Appendix for proton, iron and photon primaries. In Fig. 3, the ToT trigger probability from parametrization has been superimposed on the simulation (proton primary, all zenith angles up to $65^{\circ}$ are merged). The comparison is performed as in the following. For each simulated event, i.e. for a certain primary, energy and arrival direction, the LTP is calculated using the parametrization (lines) and shown together with the full simulation (points). The agreement is remarkably good in the entire energy range for proton (shown in the figure) and for iron and photon primaries.

\subsection{Dependence on primary mass}

The detector response to showers induced by different primary particles is shown in Fig. 4, for two classes of events, vertical $\left(0^{\circ}<\theta<38^{\circ}\right)$ on the top and moderately inclined $\left(38^{\circ}<\theta<65^{\circ}\right)$ on the bottom. Because of their larger number of muons, showers induced by iron nuclei provide a higher trigger capability at larger distances than those induced by protons, for all zenith angles. However, the difference between proton and iron is too small to give any hint for mass composition analysis. On the other hand, the LTP functions for photon primaries differ sensibly from those of hadrons (they vanish at shorter distances, about $500 \mathrm{~m}$ less at an energy of $10^{19} \mathrm{eV}$ ). This is a consequence of the structure of the lateral distribution of photon showers, i.e. at a given energy, their effective footprint at the ground is smaller than the one of hadrons. Moreover, in photon showers there is a much smaller number of muons.

It is worth noting that the energy threshold corresponding to full efficiency for SD, derived from data and simulation in Ref. [4], has been found to be compatible with the expectation for hadronic primaries.

\subsection{Dependence on hadronic interaction model}

Different choices of high energy interaction models influence the simulation of shower development and could affect the expected trigger efficiency. The dependence of the Lateral Trigger Probability on the assumptions for the hadronic interaction model has been investigated using a sample of simulated showers (proton) produced with SIBYLL [16]. As shown in Fig. 5, the LTP 

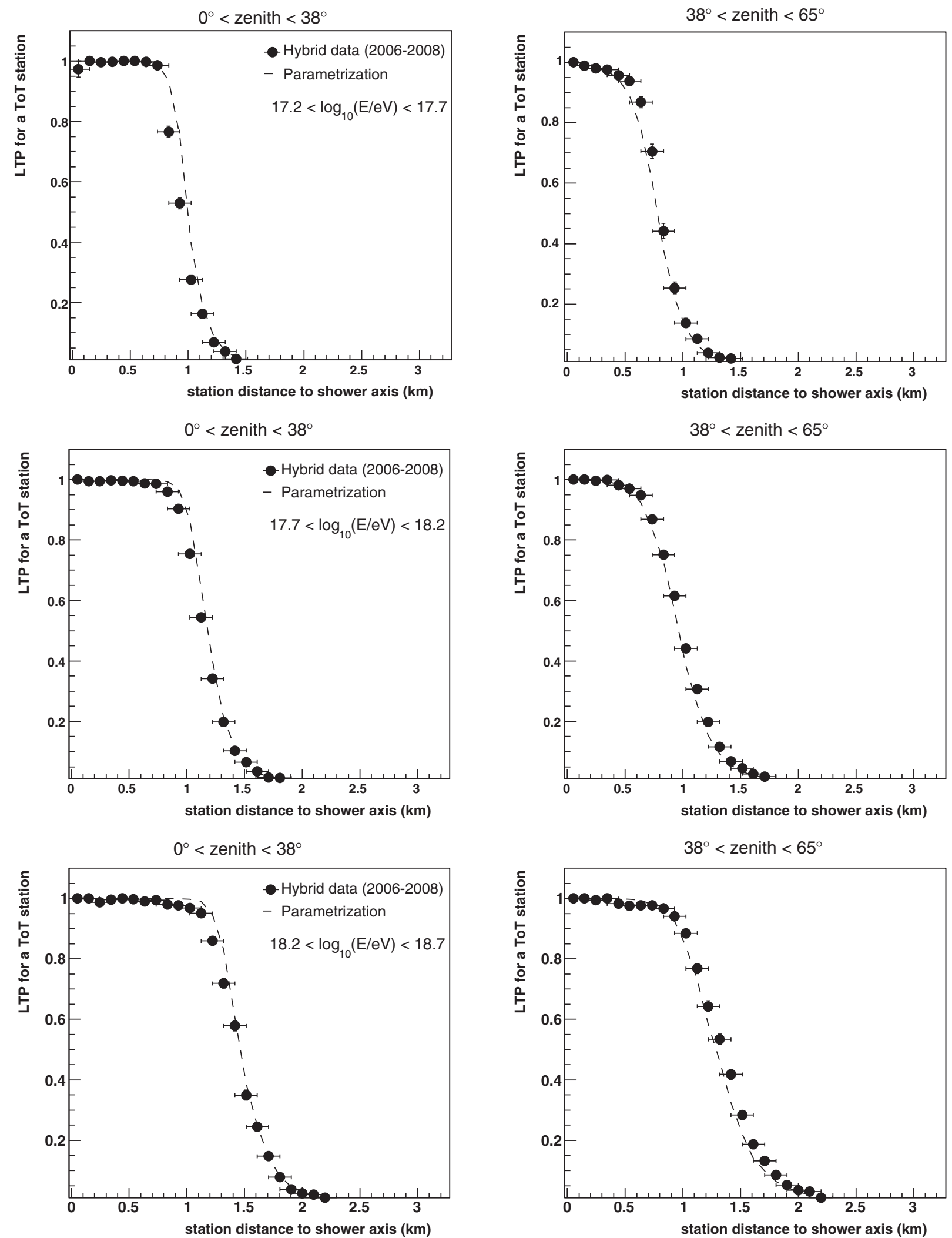

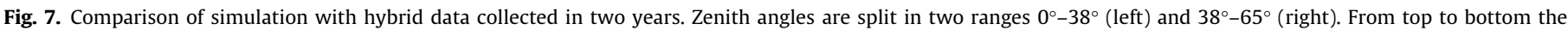
energy intervals are $10^{17.2}<E<10^{17.7} \mathrm{eV}, 10^{17.7}<E<10^{18.2} \mathrm{eV}, 10^{18.2}<E<10^{18.7} \mathrm{eV}, 10^{18.7}<E<10^{19.2} \mathrm{eV}$.

functions derived with the two hadronic interaction models differ only at large distance from the shower axis, in a range where the efficiency degrades rapidly. In this region, SIBYLL gives a lower
LTP since this model predicts on average a smaller number of muons. Those differences are however too small to imply an observable impact on the detector acceptance. 


\section{LTP functions from data and comparison with simulation}

The LTP functions can be derived from data by calculating the ratio of triggered to active stations within a given distance from the reconstructed shower axis. While doing this, the actual surface detector configuration must be accurately taken into account as a function of time. In addition, only high quality data are selected to avoid biases due to mis-reconstructed energies and/or arrival directions. The use of hybrid events allows to derive LTP functions also for energies below the threshold of an independent SD trigger. This is a benefit of the hybrid design that aims to fully exploit the distinctive potential offered by the Pierre Auger Observatory. Two years of hybrid data collected between June 2006 and May 2008 were used for this study. The events are selected as described in [3] and this ensures an angular resolution of about $0.6^{\circ}$ and a core position determination better than $70 \mathrm{~m}$. Further requirements on the goodness of the reconstructed longitudinal profile provide an energy resolution of about $10 \%$ above $10^{18} \mathrm{eV}$ and less than $15 \%$ at lower energies [3].

The LTP measured from data is shown in Fig. 6 for different energy intervals. To verify the performance of the parametrization described in Section 3, for each selected event, the LTP of any active station within $3 \mathrm{~km}$ from the shower axis is calculated using the reconstructed energy and direction. The predicted probability (dashed line) is then superimposed on data (points), see Fig. 6. In this way, data are compared to simulation taking into account the actual status of the detector. The shaded area gives the interval of expected values assuming that data are pure proton (lower edge) or pure iron (upper edge). A 50\% proton and 50\% iron mixed composition has been assumed for the parametrization (dashed line). The agreement is good over the entire energy range. This feature actually starts at very low energies, even below the range of full efficiency for the hybrid detection [3]. In this case, whereas in data only events with at least one SD ToT station are selected, in simulation also the events that did not trigger at all are taken into account in the calculation of the probability. As a consequence, the comparison between data and simulation could be biased. However, the good level of agreement actually reached reflects the fact that the hybrid detection is very close to fully efficient and the energy reconstruction remains reasonably good within the scope of this analysis down to energy of about $10^{17.5} \mathrm{eV}$.

For each energy interval considered, the agreement between data and simulation has also proven to hold in two zenith angle bands $\left(0^{\circ}-38^{\circ}\right.$ and $\left.38^{\circ}-65^{\circ}\right)$, see Fig. 7 .

\subsection{Impact of weather effect on LTP}

The effect of atmospheric variations (in pressure, temperature and air density) on extensive air showers development has been extensively studied with the surface detector data [17]. A significant modulation of the rate of events with the atmospheric variables, both on a seasonal scale $(\sim 10 \%)$ and on a shorter time scale ( $\sim 2 \%$ on average during a day) has been observed. This modulation is mainly explained as due to the change with the air density of the Molière radius near ground thus influencing the trigger probability and the rate of events above a fixed energy. Hybrid data in the energy range around $10^{18} \mathrm{eV}$ have been used to investigate this effect on LTP. Data have been separated by season and are shown, together with the parametrization, for austral winter and austral summer, see Fig. 8, top panel. The ratio of summer and winter relative to the parametrization is shown in the bottom panel. Results qualitatively match the expectation. Higher temperature at the ground, as for the austral summer, induces a reduction of the air density weakly enhancing the trigger probability at a given distance relative to all other seasons. Nevertheless the effect is almost negligible on the scale of the measurable trigger efficiency.
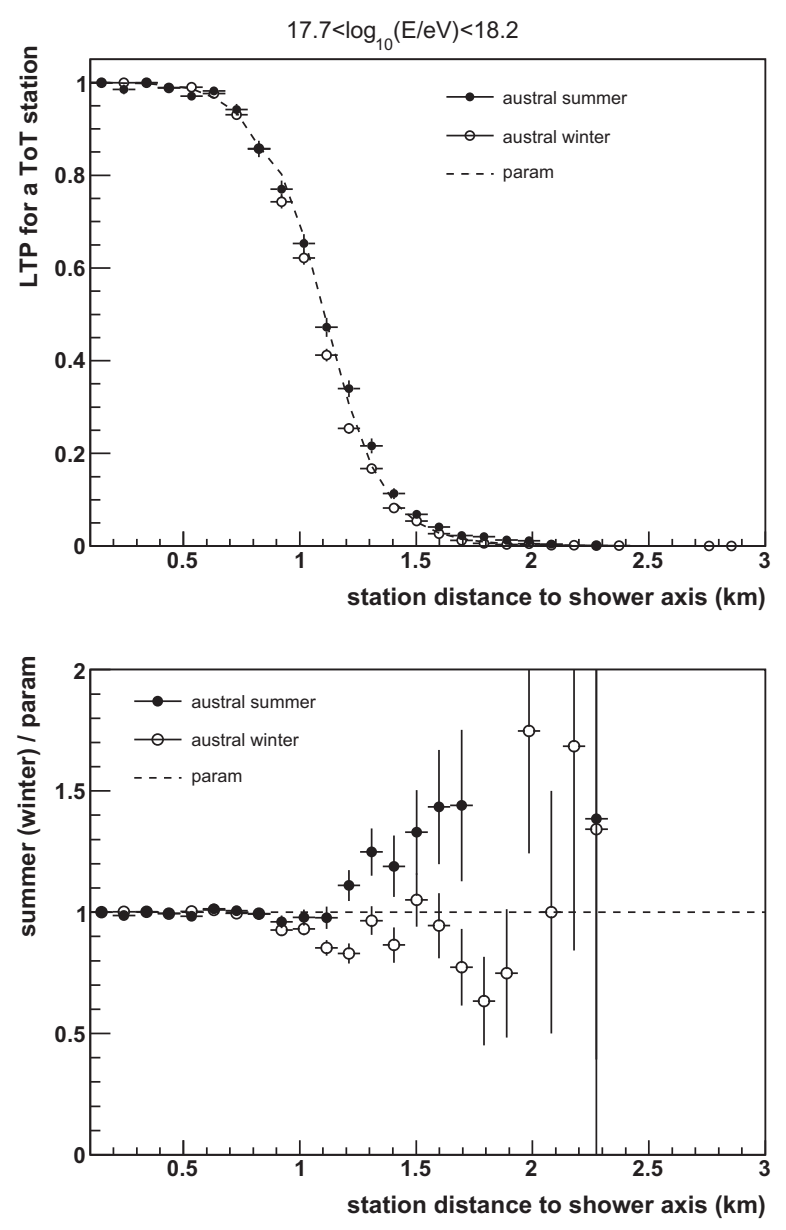

Fig. 8. LTP functions from hybrid data at energy of about $10^{18} \mathrm{eV}$ for austral winter and austral summer compared to the parametrization derived in Section 3 (top) and ratio relative to the parametrization (bottom).

\section{Summary and Conclusions}

In the previous sections we have introduced the concept of Lateral Trigger Probability function as a tool to characterize the single detector trigger efficiency. We have derived LTP functions for the particular case of the surface detector of the Pierre Auger Observatory using simulations. We discussed their evolution with different physical parameters of air showers such as the energy, zenith angle and nature of the primary particle. We also investigated the impact of choosing different hadronic interaction models in the simulations. Furthermore, we estimated the LTP functions at different energies and zenith angles using hybrid data and showed that seasonal effects are visible in the trigger probabilities retrieved from data as expected from previous studies [17].

The good agreement between simulations and data over a wide energy range (between $10^{17.5} \mathrm{eV}$ and $10^{19} \mathrm{eV}$ ) demonstrates the accuracy of the different aspects of the simulation procedure (i.e. air shower, detectors and trigger simulation) as well as the quality of the reconstruction obtained for hybrid data. These comparisons support and validate the use of simulated LTP functions in the estimate of the hybrid aperture described in [3]. Monitoring the LTP functions over a longer period of time can be used to study the long-term performance of the SD trigger for individual stations both above and below the acceptance saturation energy.

As a final consideration, LTP functions can be derived at higher energies using SD-only data because, at energy above $\sim 10^{18.5} \mathrm{eV}$, despite the statistics of hybrids becoming small, the surface detector is fully efficient and the geometrical reconstruction is accurate. 
As mentioned in the Introduction, the probability of a high level trigger for the surface detector is a combination of single detector probabilities. Hence LTP functions provide a robust and simple method to estimate the energy or zenith angle dependence of SD acceptance for any arbitrary configuration. This makes this technique a valuable tool to design other experiments and future enhancements of the Pierre Auger Observatory.

\section{Acknowledgements}

The successful installation and commissioning of the Pierre Auger Observatory would not have been possible without the strong commitment and effort from the technical and administrative staff in Malargüe.

We are very grateful to the following agencies and organizations for financial support: Comisión Nacional de Energía Atómica, Fundación Antorchas, Gobierno De La Provincia de Mendoza, Municipalidad de Malargüe, NDM Holdings and Valle Las Leñas, in gratitude for their continuing cooperation over land access, Argentina; the Australian Research Council; Conselho Nacional de Desenvolvimento Científico e Tecnológico (CNPq), Financiadora de Estudos e Projetos (FINEP), Fundação de Amparo à Pesquisa do Estado de Rio de Janeiro (FAPERJ), Fundação de Amparo à Pesquisa do Estado de São Paulo (FAPESP), Ministério de Ciência e Tecnologia (MCT), Brazil; AVCR, AV0Z10100502 and AV0Z10100522, GAAV KJB300100801 and KJB100100904, MSMT-CR LA08016, LC527, 1M06002, and MSM0021620859, Czech Republic; Centre de Calcul IN2P3/CNRS, Centre National de la Recherche Scientifique (CNRS), Conseil Régional Ile-de-France, Département Physique Nucléaire et Corpusculaire (PNC-IN2P3/CNRS), Département Sciences de l'Univers (SDU-INSU/CNRS), France; Bundesministerium für Bildung und Forschung (BMBF), Deutsche Forschungsgemeinschaft

(DFG), Finanzministerium Baden-Württemberg, HelmholtzGemeinschaft Deutscher Forschungszentren (HGF), Ministerium für Innovation, Wissenschaft und Forschung, Nordrhein-Westfalen, Ministerium für Wissenschaft, Forschung und Kunst, Baden-Württemberg, Germany; Istituto Nazionale di Fisica Nucleare (INFN), Istituto Nazionale di Astrofisica (INAF), Ministero dell'Istruzione, dell'Università e della Ricerca (MIUR), Gran Sasso Center for Astroparticle Physics (CFA), Italy; Consejo Nacional de Ciencia y Tecnología (CONACYT), Mexico; Ministerie van Onderwijs, Cultuur en Wetenschap, Nederlandse Organisatie voor Wetenschappelijk Onderzoek(NWO), Stichting voor Fundamenteel Onderzoek der Materie (FOM), Netherlands; Ministry of Science and Higher Education, Grant Nos. 1 P03 D 01430 and N N202 207238, Poland; Fundação para a Ciência e a Tecnologia, Portugal; Ministry for Higher Education, Science, and Technology, Slovenian Research Agency, Slovenia; Comunidad de Madrid, Consejería de Educación de la Comunidad de Castilla La Mancha, FEDER funds, Ministerio de Ciencia e Innovación and Consolider-Ingenio 2010 (CPAN), Generalitat Valenciana, Junta deAndalucía, Xunta deGalicia, Spain; Science and Technology FacilitiesCouncil, United Kingdom; Department ofEnergy, Contract Nos. DEAC02-07CH11359, DE-FR02-04ER41300, National Science Foundation, Grant No. 0969400, The Grainger Foundation USA; NAFOSTED, VietNam; ALFA-EC/ HELEN, European Union 6th Framework Program, Grant No. MEIF-CT-2005-025057, European Union 7th Framework Program, Grant No. PIEF-GA-2008-220240, and UNESCO.

\section{Appendix A. LTP parametrization}

The LTP is fitted, as discussed in Section 3, to the following function:

$\operatorname{LTP}(r)= \begin{cases}\frac{1}{1+e^{\frac{r-R_{0}}{\Delta R}}} & r \leqslant R_{0} \\ \frac{1}{2} e^{C \cdot\left(r-R_{0}\right)} & r>R_{0}\end{cases}$

with $R_{0}$ being the distance where the LTP is equal to 0.5 .
The dependences of fit parameters $R_{0}, \Delta R$ and $C$ on energy and zenith angle can be parametrized by quadratic polynomials in the variables $\cos \theta$ and $\log _{10}(E / \mathrm{eV})$. The corresponding coefficients are given for proton, iron and photon primaries $\left(0^{\circ}<\theta<65^{\circ}\right)$, separately. Concerning the accuracy of the parameters, a change at the level of $(1 \div 5) \%$ propagates approximately linearly in the returned value of the parametrization.

\section{A.1. Proton showers}

The overall parametrization for proton primaries $\left(0^{\circ}<\theta<65^{\circ}\right)$ is summarized in the following matrix equation:

$$
\begin{aligned}
& \frac{R_{0}}{\mathrm{~km}}=\left(\begin{array}{c}
1 \\
\cos \theta \\
\cos ^{2} \theta
\end{array}\right)^{T} \cdot\left[\left(\begin{array}{ccc}
4.30 \cdot 10^{1} & -6.21 \cdot 10^{0} & 2.09 \cdot 10^{-1} \\
-9.89 \cdot 10^{0} & 3.22 \cdot 10^{0} & -1.34 \cdot 10^{-1} \\
-8.24 \cdot 10^{0} & -2.29 \cdot 10^{-1} & 3.11 \cdot 10^{-2}
\end{array}\right)\right. \\
& \left.\left(\begin{array}{c}
1 \\
\log _{10}(E / e V) \\
\log _{10}^{2}(E / e V)
\end{array}\right)\right] \\
& \frac{\Delta R}{\mathrm{~km}}=\left(\begin{array}{c}
1 \\
\cos \theta \\
\cos ^{2} \theta
\end{array}\right)^{T} \cdot\left[\left(\begin{array}{ccc}
-3.90 \cdot 10^{0} & 4.38 \cdot 10^{-1} & -1.15 \cdot 10^{-2} \\
1.19 \cdot 10^{1} & -1.37 \cdot 10^{0} & 3.82 \cdot 10^{-2} \\
-6.19 \cdot 10^{0} & 7.14 \cdot 10^{-1} & -1.99 \cdot 10^{-2}
\end{array}\right)\right. \\
& \left.\left(\begin{array}{c}
1 \\
\log _{10}(E / e V) \\
\log _{10}^{2}(E / e V)
\end{array}\right)\right] \\
& \frac{C}{\mathrm{~km}^{-1}}=\left(\begin{array}{c}
1 \\
\cos \theta \\
\cos ^{2} \theta
\end{array}\right)^{T} \cdot\left[\left(\begin{array}{ccc}
-3.28 \cdot 10^{2} & 3.48 \cdot 10^{1} & -9.16 \cdot 10^{-1} \\
-4.37 \cdot 10^{1} & 3.96 \cdot 10^{0} & -1.10 \cdot 10^{-1} \\
0 & 0 & 0
\end{array}\right)\right. \\
& \left.\cdot\left(\begin{array}{c}
1 \\
\log _{10}(E / e V) \\
\log _{10}^{2}(E / e V)
\end{array}\right)\right]
\end{aligned}
$$

\section{A.2. Iron showers}

The overall parametrization for iron primaries $\left(0^{\circ}<\theta<65^{\circ}\right)$ is summarized in the following matrix equation:

$$
\begin{aligned}
\frac{R_{0}}{\mathrm{~km}}= & \left(\begin{array}{c}
1 \\
\cos \theta \\
\cos ^{2} \theta
\end{array}\right)^{T} \cdot\left[\left(\begin{array}{ccc}
4.90 \cdot 10^{1} & -6.97 \cdot 10^{0} & 2.33 \cdot 10^{-1} \\
-9.23 \cdot 10^{3} & 3.07 \cdot 10^{0} & -1.30 \cdot 10^{-1} \\
-24.4 \cdot 10^{3} & 1.69 \cdot 10^{0} & -2.43 \cdot 10^{-2}
\end{array}\right)\right. \\
& \left.\cdot\left(\begin{array}{c}
1 \\
\log _{10}(E / \mathrm{eV}) \\
\log _{10}^{2}(\mathrm{E} / \mathrm{eV})
\end{array}\right)\right]
\end{aligned}
$$$$
\begin{aligned}
\frac{\Delta R}{\mathrm{~km}}= & \left(\begin{array}{c}
1 \\
\cos \theta \\
\cos ^{2} \theta
\end{array}\right)^{T} \cdot\left[\left(\begin{array}{ccc}
-9.52 \cdot 10^{-1} & 6.81 \cdot 10^{-2} & 0 \\
1.46 \cdot 10^{0} & -1.04 \cdot 10^{-1} & 0 \\
-9.32 \cdot 10^{-1} & 6.36 \cdot 10^{-2} & 0
\end{array}\right)\right. \\
& \left.\cdot\left(\begin{array}{c}
1 \\
\log _{10}(E / \mathrm{eV}) \\
\log _{10}^{2}(E / \mathrm{eV})
\end{array}\right)\right]
\end{aligned}
$$ 


$$
\begin{aligned}
\frac{C}{\mathrm{~km}^{-1}}= & \left(\begin{array}{c}
1 \\
\cos \theta \\
\cos ^{2} \theta
\end{array}\right)^{T} \cdot\left[\left(\begin{array}{ccc}
-8.82 \cdot 10^{2} & 9.50 \cdot 10^{1} & -2.56 \cdot 10^{0} \\
3.83 \cdot 10^{2} & -4.40 \cdot 10^{1} & 1.24 \cdot 10^{0} \\
0 & 0 & 0
\end{array}\right)\right. \\
& \left.\cdot\left(\begin{array}{c}
1 \\
\log _{10}(E / \mathrm{eV}) \\
\log _{10}^{2}(\mathrm{E} / \mathrm{eV})
\end{array}\right)\right]
\end{aligned}
$$

\section{A.3. Photon showers}

The overall parametrization for photon primaries $\left(0^{\circ}<\theta<65^{\circ}\right)$ is summarized in the following matrix equation:

$$
\begin{aligned}
\frac{R_{0}}{\mathrm{~km}}= & \left(\begin{array}{c}
1 \\
\cos \theta \\
\cos ^{2} \theta
\end{array}\right)^{T} \cdot\left[\left(\begin{array}{ccc}
1.07 \cdot 10^{2} & -1.31 \cdot 10^{1} & 3.89 \cdot 10^{-1} \\
-2.46 \cdot 10^{2} & 2.90 \cdot 10^{1} & -8.30 \cdot 10^{-1} \\
1.47 \cdot 10^{2} & -1.70 \cdot 10^{1} & 4.78 \cdot 10^{-1}
\end{array}\right)\right. \\
& \left.\cdot\left(\begin{array}{c}
1 \\
\log _{10}(E / \mathrm{eV}) \\
\log _{10}^{2}(\mathrm{E} / \mathrm{eV})
\end{array}\right)\right]
\end{aligned}
$$$$
\begin{aligned}
\frac{\Delta R}{\mathrm{~km}}= & \left(\begin{array}{c}
1 \\
\cos \theta \\
\cos ^{2} \theta
\end{array}\right)^{T} \cdot\left[\left(\begin{array}{ccc}
9.03 \cdot 10^{0} & -1.02 \cdot 10^{0} & 3.05 \cdot 10^{-2} \\
-2.76 \cdot 10^{1} & 3.15 \cdot 10^{0} & -9.26 \cdot 10^{-2} \\
2.46 \cdot 10^{1} & -2.82 \cdot 10^{0} & 8.25 \cdot 10^{-2}
\end{array}\right)\right. \\
& \left.\cdot\left(\begin{array}{c}
1 \\
\log _{10}(E / e V) \\
\log _{10}^{2}(E / e V)
\end{array}\right)\right]
\end{aligned}
$$

$$
\begin{aligned}
\frac{C}{\mathrm{~km}^{-1}}= & \left(\begin{array}{c}
1 \\
\cos \theta \\
\cos ^{2} \theta
\end{array}\right)^{T} \cdot\left[\left(\begin{array}{ccc}
-9.34 \cdot 10^{3} & 1.04 \cdot 10^{3} & -2.91 \cdot 10^{1} \\
2.60 \cdot 10^{4} & -2.91 \cdot 10^{3} & 8.10 \cdot 10^{1} \\
-1.67 \cdot 10^{4} & 1.86 \cdot 10^{3} & -5.17 \cdot 10^{1}
\end{array}\right)\right. \\
& \left.\cdot\left(\begin{array}{c}
1 \\
\log _{10}(E / \mathrm{eV}) \\
\log _{10}^{2}(\mathrm{E} / \mathrm{eV})
\end{array}\right)\right]
\end{aligned}
$$

\section{References}

[1] J. Abraham et al., Pierre Auger Collaboration, Nuclear Instruments and Methods in Physics Research A523 (2004) 50.

[2] J. Abraham et al., Pierre Auger Collaboration, Nuclear Instruments and Methods in Physics Research A620 (2010) 227.

[3] P. Abreu et al., Pierre Auger Collaboration, Astroparticle Physics 34 (2011) 368

[4] J. Abraham et al., Pierre Auger Collaboration, Nuclear Instruments and Methods in Physics Research A613 (2010) 29.

[5] J. Abraham et al., Pierre Auger Collaboration, Physics Letters B685 (2010) 239

[6] J. Abraham et al., Pierre Auger Collaboration, Physical Review Letters 104 (2010) 091101.

[7] D. Allard, in: 29th International Cosmic Ray Conference (2005) <arxiv:astro$\mathrm{ph} / 0511104 \mathrm{v} 1>$.

[8] D. Heck et al., CORSIKA: a Monte Carlo Code to Simulate Extensive Air Showers, Report FZKA 6019, (1998).

[9] D. Heck and J. Knapp, Report FZKA 6097 (1998) Forschungszentrum Karlsruhe; <http://www-ik.fzk.de/heck/publications/>.

[10] S. Ostapchenko, Physics Letters B636 (2006) 40; S. Ostapchenko, Physical Review D74 (2006) 014026

[11] A. Fassò et al. FLUKA: a multi-particle transport code, CERN-2005-10 (2005) INFN/TC_05/11, SLAC-R-773; A. Fassò et al. The physics models of FLUKA: status and recent developments, in: Computing in High Energy and Nuclear Physics 2003 Conference (CHEP2003), La Jolla, CA, USA, March 24-28, 2003, (paper MOMT005) eConf C0303241 (2003) <arxiv:hep-ph/0306267>.

[12] S. Agostinelli et al., Nuclear Instruments and Methods in Physics Research A506 (2003) 250;

S. Agostinelli et al., IEEE Transactions on Nuclear Science 53 (1) (2006) 270.

[13] P. Billoir, Astroparticle Physics 30 (2008) 270.

[14] S. Argirò et al., Nuclear Instruments and Methods in Physics Research A580 (2007) 1485.

[15] M.T. Dova, M.E. Manceido, A.G. Mariazzi, H. Wahlberg, F. Arqueros, D. GarcaPinto, Astroparticle Physics 31 (2009) 312.

[16] Eun-Joo Ahn et al., Physical Review D80 (2009) 094003; R.S. Fletcher et al., Physical Review D50 (1994) 5710.

[17] J. Abraham et al., Pierre Auger Collaboration, Astroparticle Physics 32 (2009) 89. 